\title{
Pathological complete response by advanced hepatocellular carcinoma with massive macrovascular invasion to hepatic arterial infusion chemotherapy: a case report
}

\author{
Shusei Sano ${ }^{1 *}$, Shinji Nakata', Shuichi Wada², Masatsugu Kuroiwa ${ }^{1}$, Hiroki Sakai ${ }^{1}$, Kei Kusama' ${ }^{1}$ Taiichi Machida', \\ Akihito Nishio ${ }^{1}$, Ichiro Ito ${ }^{3}$ and Harutsugu Sodeyama'
}

\begin{abstract}
Background: Advanced hepatocellular carcinoma (HCC) with macrovascular invasion has an extremely dismal prognosis. We report a rare case of multiple HCC with tumor thrombosis in the portal vein and inferior vena cava that was initially treated with hepatic arterial infusion chemotherapy (HAIC); later resection revealed pathological complete response.

Case presentation: A 75-year-old man presented with HCC in his right liver, with tumor thrombosis growing to the right portal vein and the inferior vena cava, and bilateral intrahepatic liver metastases. He underwent HAIC (5fluorouracil $\left[170 \mathrm{mg} / \mathrm{m}^{2}\right]+$ cisplatin $\left[7 \mathrm{mg} / \mathrm{m}^{2}\right]$ ) via an indwelling port. Although the tumor shrank and tumor marker levels decreased rapidly, we abandoned HAIC after one cycle because of cytopenia. We resumed HAIC 18 months later because of tumor progression, using biweekly 5-fluorouracil only [1000 mg] due to renal dysfunction. However, after 54 months, the HAIC indwelling port was occluded. The patient therefore underwent a right hepatectomy to resect the residual lesion. Histopathological findings showed complete necrosis with no viable tumor cells. The patient has been doing well without postoperative adjuvant therapy for more than 10 years after initially introducing HAIC and 6 years after the resection, without evidence of tumor recurrence.
\end{abstract}

Conclusions: HAIC can be an effective alternative treatment for advanced HCC with macrovascular invasion.

Keywords: Hepatocellular carcinoma, Macrovascular invasion, Hepatic arterial infusion chemotherapy, Complete response

\section{Background}

Advanced hepatocellular carcinoma (HCC) with macrovascular invasion has an extremely poor prognosis, with a reported median survival time (MST) of 2.7-3.1 months if left untreated $[1,2]$. In global guidelines, HCC with portal vein tumor thrombosis (PVTT) or inferior vena cava tumor thrombosis (IVCTT) is classified as the advanced stage, for which only systemic chemotherapy is

\footnotetext{
* Correspondence: sh.sano@nagano-med.jrc.or.jp

${ }^{1}$ Department of Gastroenterological Surgery, Nagano Red Cross Hospital,

5-22-1, Wakasato, Nagano-shi, Nagano 380-8582, Japan

Full list of author information is available at the end of the article
}

recommended, even in patients with good liver function $[3,4]$. Sorafenib is the standard of care for Child-Pugh A advanced HCC with macrovascular invasion and/or extrahepatic metastasis, and it significantly improved overall survival compared with supportive care [4-6]. However, MST for patients with sorafenib-treated HCC with macrovascular invasion is still poor, reportedly only 8.1 months [7]. Lenvatinib was recently shown to be non-inferior to sorafenib as first-line treatment [8], but therapeutic options are very limited for advanced HCC.

In Eastern Asian countries, various treatment options are available for HCC with macrovascular invasion,

(c) The Author(s). 2019 Open Access This article is distributed under the terms of the Creative Commons Attribution 4.0 International License (http://creativecommons.org/licenses/by/4.0/), which permits unrestricted use, distribution, and 
including systemic chemotherapy, hepatic arterial infusion chemotherapy (HAIC), transcatheter arterial chemoembolization, and surgery. Treatments are selected individually, depending on the extent of tumor thrombosis, degree of underlying cirrhosis, and patient's performance status, which can affect prognosis. However, no guidelines clarify a preferred non-operative treatment based on available evidence. We herein report a patient with multiple HCC with PVTT and IVCTT who survived after HAIC, followed by a resection that showed pathological complete response (CR) and tumor-free status for more than 6 years.

\section{Case presentation}

In February 2009, a 75-year-old man with a history of alcoholic liver disease was referred to our hospital for evaluation of multiple liver masses on abdominal ultrasound sonography. The patient had no history of hepatitis of B or $\mathrm{C}$ infection. Abdominal enhanced computed tomography (CT) showed 13-cm hypovascular liver tumors (Fig. 1a, b), with marked tumor thrombosis growing to the right portal vein (Fig. 1c) and inferior vena cava (Fig. 1d), and bilateral intrahepatic liver metastases (Fig. 1c). Serum alphafetoprotein (AFP) level and protein induced by vitamin $\mathrm{K}$ absence or antagonist-II (PIVKA-II), also known as desgamma-carboxyprothrombin, level were $3565 \mathrm{ng} / \mathrm{ml}$ and 49,000 mAU/ml, respectively. Chest CT scan, upper gastrointestinal endoscopy, and colonoscopy showed no other tumors. His carcinoembryonic antigen and carbohydrate antigen 19-9 levels were in the normal range.
Under the diagnosis of unresectable advanced HCC, an indwelling port was inserted, and HAIC with 5fluorouracil $\left(5-\mathrm{FU}, 170 \mathrm{mg} / \mathrm{m}^{2}\right)$ and cisplatin $\left(7 \mathrm{mg} / \mathrm{m}^{2}\right)$ continuously on days $1-5$ via an implanted catheter system was administered. One cycle of HAIC consisted of 5 days of treatment and 2 days rest per week for 4 consecutive weeks. Despite significant decrease in tumor markers and remarkable regression of intrahepatic lesions, PVTT, and IVCTT on enhanced CT after one HAIC cycle (Fig. 2a), we abandoned this treatment due to leukopenia and thrombocytopenia. Eight months later, when his AFP elevated to $202 \mathrm{ng} / \mathrm{ml}$, the patient refused our recommendation of sorafenib, which had become available in Japan at that year. After 18 months, during which the tumor remained silent and he was followed closely without treatment (Fig. 2b), his AFP and PIVKA-II levels rapidly elevated to $21,490 \mathrm{ng} / \mathrm{ml}$ and $1444 \mathrm{mAU} / \mathrm{ml}$ (respectively), and enhanced CT showed tumor progression (Fig. 2c). Therefore, we resumed the HAIC at the same dose for one cycle, but switched to 5-FU alone (1000 mg biweekly) due to renal dysfunction. Twenty-one months after resuming HAIC, we stopped this treatment because the indwelling port became occluded. At that time, the patient's serum AFP and PIVKA-II were within normal ranges, and enhanced $\mathrm{CT}$ and magnetic resonance images indicated that the tumor was still shrunken with necrotic areas, and showed no PVTT, IVCTT, or intrahepatic metastases (Fig. 3). He had good hepatic function (Child-Pugh classification A5 and liver damage A) with atrophy of the
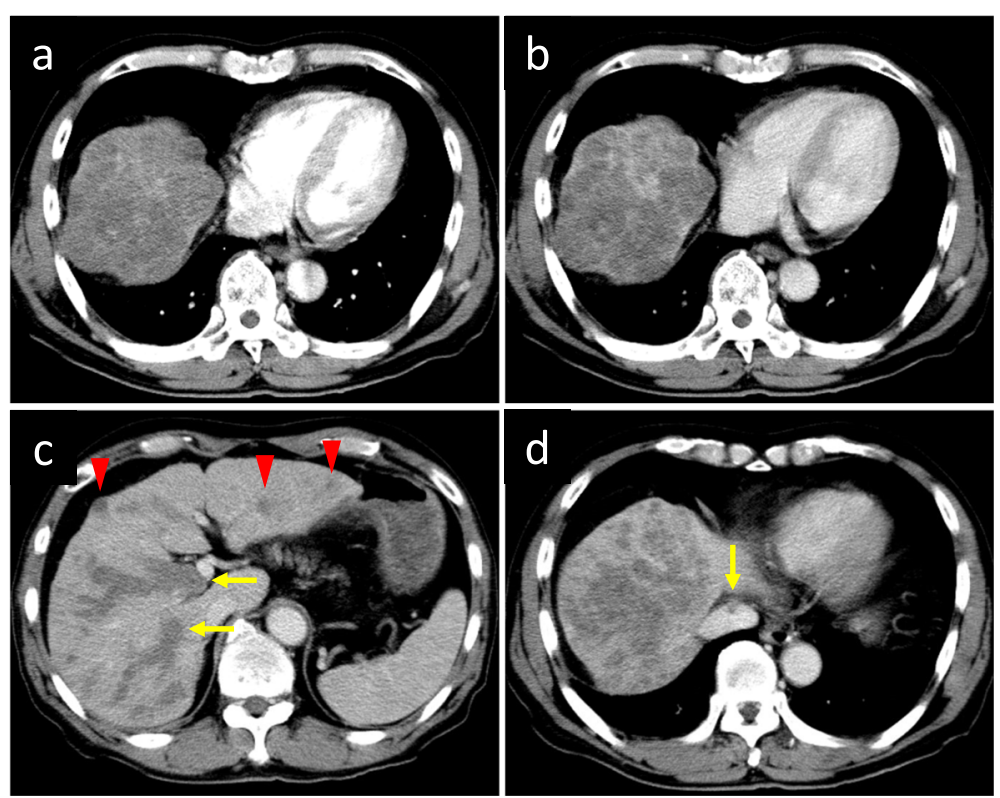

Fig. 1 Enhanced CT images before introducing HAIC. Heterogeneous, 13-cm mass in the right liver shows hypovascular appearance in the arterial phase (a) and in the portal phase (b). Massive tumor thrombosis (arrow) growing to the right portal vein (c) and inferior vena cava (d), with bilateral intrahepatic liver metastases (arrowhead) (c). CT, computed tomography; HAIC, hepatic arterial infusion chemotherapy 

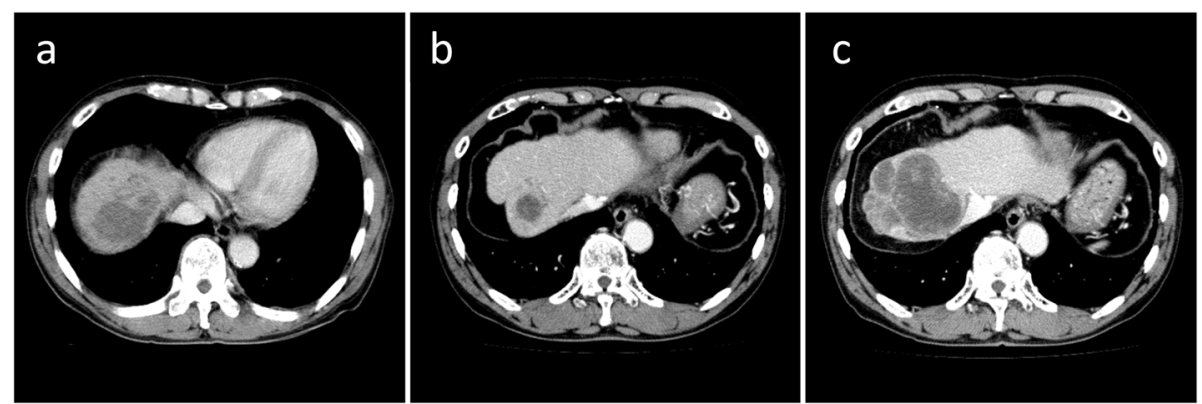

Fig. 2 Enhanced CT images after introducing HAIC. Remarkable regression of intrahepatic lesions, thrombosis in the right portal vein and inferior vena cava is seen after one HAIC cycle (a). Tumor remained shrunk without treatment for 16 months after interrupting HAIC (b). Tumor progression occurred 18 months after the HAIC interruption (c)

right hepatic lobe $(131 \mathrm{ml}$, corresponding to $15.1 \%$ of liver volume), despite indocyanine green retention rate being $15.0 \%$. We therefore performed a right hepatic lobectomy to remove the residual lesion, at 54 months after his initial treatment. He was discharged on postoperative day 14 without postoperative complications.

The resected specimen showed the solid tumor with significant hemorrhage and necrosis (Fig. 4a, b). Microscopic examination revealed a nodule with a central necrotic core, surrounded by a thick hyalinized fibrotic capsule (Fig. 4c, d). No residual viable tumor cells were observed (Fig. 4d, e). Bilirubin pigments surrounded by necrotic tissue in the central necrotic compartment indicated that the tumor was HCC (Fig. 4d). No adjuvant therapy was performed. CT imaging has shown no signs of recurrence, and his tumor markers have also been within the normal limits for the past 126 and 72 months after the initial HAIC and after the operation, respectively. His clinical course is summarized in Fig. 5.

\section{Discussion}

The present case shows the effectiveness of HAIC for advanced $\mathrm{HCC}$ with multiple intrahepatic metastases, PVTT, and IVCTT. A CR was pathologically proven after conversion surgery. We believe that HAIC would be the main cause of complete remission in this patient because of the following reasons: First, the patient did not receive medical care other than HAIC. Second, tumor regression completely coincided with the timing of HAIC. Tumor shrinkage and decrease in tumor markers were observed only when he received HAIC. Although CR in advanced HCC patients with macrovascular invasion has been
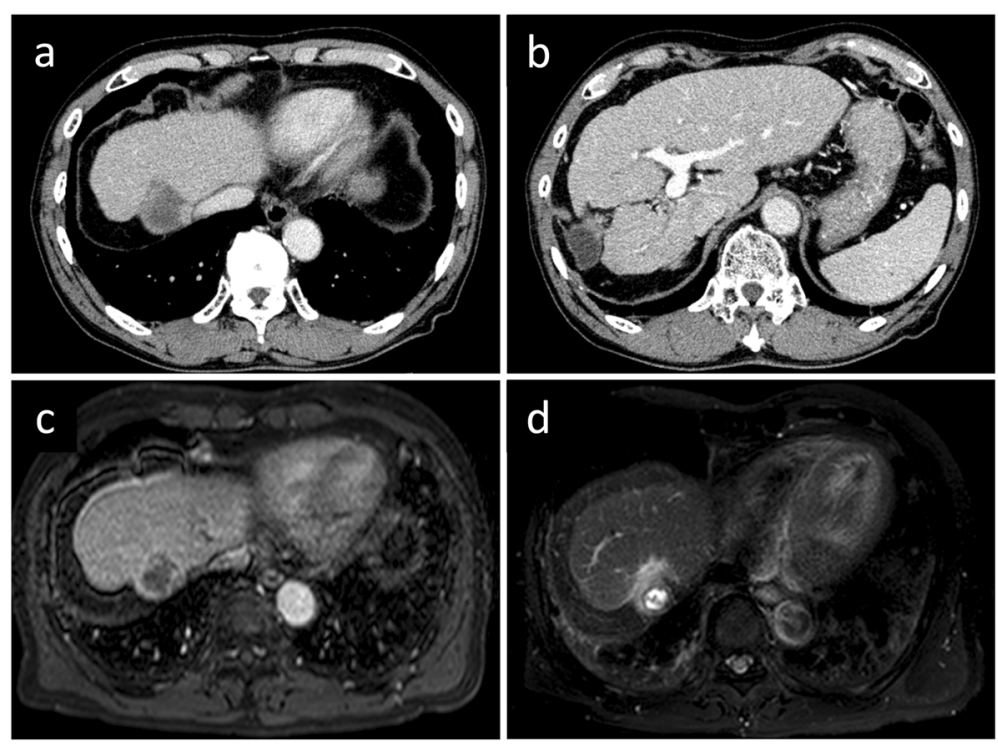

Fig. 3 Enhanced CT and magnetic resonance imaging images before hepatectomy (54 months after the initial HAIC). Persistent tumor shrinkage with necrotic area, and no tumor thrombosis in the portal vein or the inferior vena cava, or intrahepatic metastases (a, b). Intratumoral necrotic area in the portal phase (c) and diffusion-weighted imaging (d) 


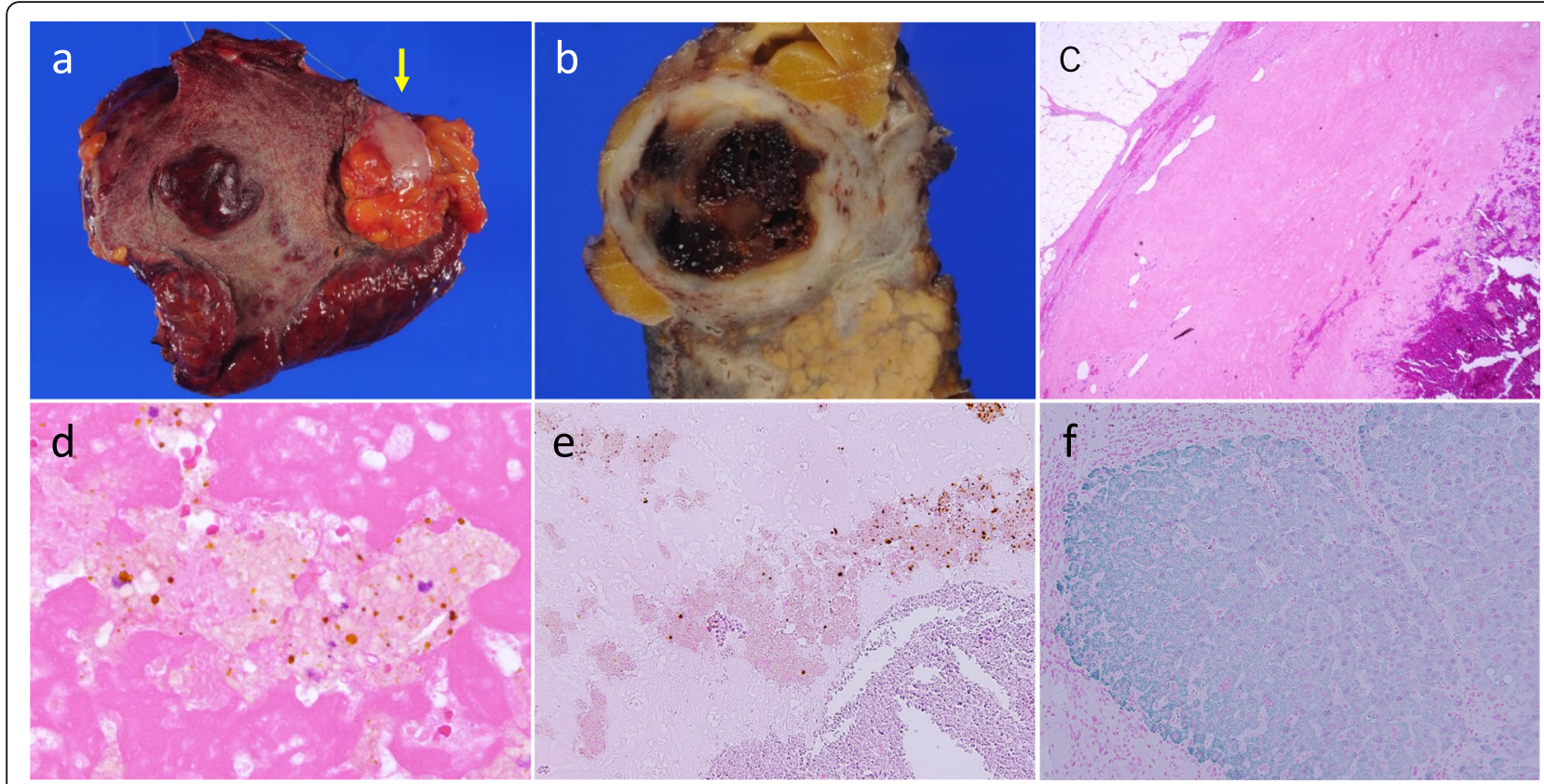

Fig. 4 Gross and histopathological findings of the resected specimen. Whitish tumor surrounded by omentum at the liver surface (arrow). Background liver was composed of geographical atrophic area and cirrhotic liver parenchyma (a). The cut surface of the tumor shows the solid tumor with significant hemorrhage and necrosis (b). Microscopic finding of the liver mass shows complete necrosis surrounded by a thick hyalinized fibrotic capsule without any viable tumor cells (hematoxylin-eosin staining, $\times 40$ ) (c). Bilirubin pigments surrounded by necrotic tissue in the central necrotic area (hematoxylin-eosin staining, $\times 400)(\mathbf{d})$. Immunohistochemical staining with hepatocyte specific antigen antibody shows nucleated cells in the tumor are negatively stained $(\times 200)(\mathbf{e})$, while green dye positive on non-tumorous hepatocyte $(\times 200)(\mathbf{f})$

previously described, most of these cases were treated with sorafenib alone $[9,10]$ or sorafenib combined therapy [11-16]. Only four reports written in English have shown a CR from HAIC in patients with advanced HCC [17-20]. Therefore, the present case was a rare case of $\mathrm{CR}$ achieved by HAIC alone, leading to a curative surgical resection following overall survival of more than 6 years without any adjuvant treatment.

HAIC uses high concentrations of anticancer agents administered directly into the hepatic artery via an injection port. It can enhance efficacy of drugs by localizing their application and minimizing systemic adverse effects. HAIC is frequently used against advanced HCC with macrovascular invasion in Eastern Asian patients. Among several HAIC protocols, the combination of 5$\mathrm{FU}$ and cisplatin is one of the most common therapeutic regimens although there are slight differences of dose and duration setting in each study; a high response rate of $31-48 \%$ and improved MST of 14.0-31.6 months have been reported [2, 21-24]. Several studies have shown the efficacy of HAIC compared with sorafenib for advanced $\mathrm{HCC}$ with macrovascular invasion. Moriguchi et al., in a study of severe tumor thrombus in the first branches of the portal vein and/or the main portal vein, found MST (10.1 vs. 3.9 months) and median time-totreatment-failure (3.5 vs. 1.2 months) were significantly longer in the HAIC with 5-FU and cisplatin group than in the sorafenib group [24]. Nakano et al. reported a prospective cohort study in which the therapeutic response rate of HAIC using cisplatin suspension in Lipiodol combined with 5-FU (New FP) was superior to that of sorafenib; median overall survival for the New FP and sorafenib groups was 30.4 and 13.2 months, respectively $(P=0.013)$ [25]. Kudo et al. reported that adding HAIC with 5-FU and cisplatin to sorafenib might improve overall survival in HCC patients with main portal vein invasion (11.4 vs. 6.5 months) [26]. While its benefits have not been confirmed in a randomized control study, HAIC with 5-FU and cisplatin may offer a better response to treatment than sorafenib in advanced HCC patients with massive macrovascular invasion.

It is controversial whether duration of HAIC reflects therapeutic effect. In the previous reports that described pathological CR by HAIC alone, the treatment periods ranged from 3 to 26 months [17-20]. In the present case, tumor progression was observed after regression following a single cycle of initial HAIC, which suggests that the treatment period was too short. Following longterm HAIC for 21 months would control the tumor and lead to the complete remission. Based on the fact that the present HCC showed a hypovascular appearance, tumor vascularity might also relate to the tumor shrinkage. HCC tends to appear hypovascular and heterogeneous on contrast-enhanced CT if an HCC patient has a 


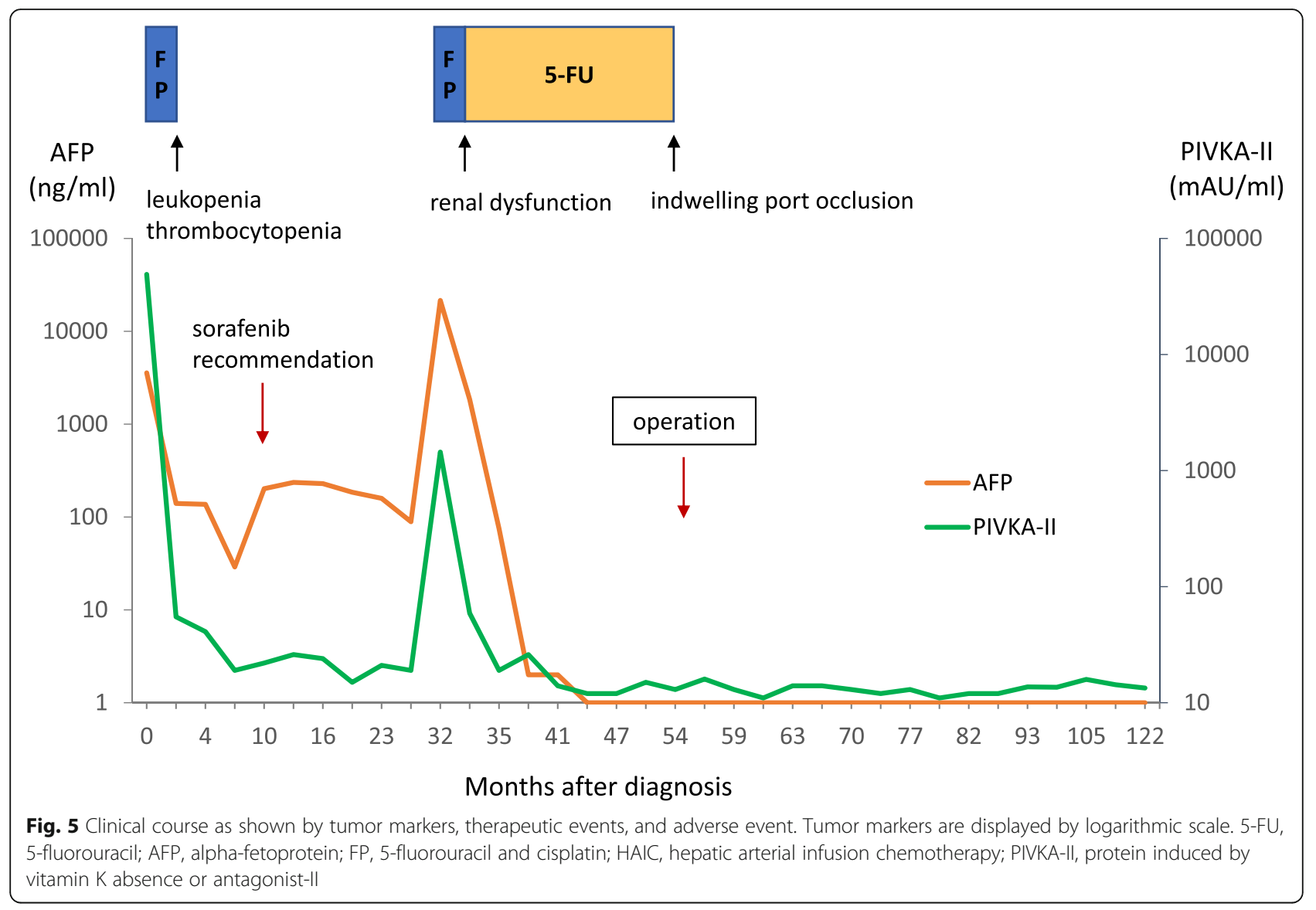

high level of serum vascular endothelial growth factor (VEGF) [27]. Abnormal tumor vascular networks induced by VEGF develop tumor hypoxia: an important factor of spontaneous tumor regression [28, 29]. Thus, hypovascular appearance as well as long-term HAIC would contribute to the complete remission in the present case. Prognosis of non-responders to HAIC was known to be poor, and remarkable responses as in the present case are rare and challenging. Therefore, establishment of a pretherapeutic assessment of candidates for HAIC is needed to provide optimal treatment to patients with advanced HCC.

\section{Conclusion}

Even though only systemic chemotherapy has been approved worldwide for patients with advanced unresectable HCC, the present case suggests HAIC has been effective and can be an alternative treatment option for advanced HCC with macrovascular invasion.

\section{Abbreviations}

5-FU: 5-Fluorouracil; AFP: Alpha-fetoprotein; CT: Computed tomography; HAIC: Hepatic arterial infusion chemotherapy; HCC: Hepatocellular carcinoma; IVCTT: Inferior vena cava tumor thrombosis; MST: Median survival time; PIVKA-II: Protein induced by vitamin K absence or antagonist-II; PVIT: Portal vein tumor thrombosis

\section{Acknowledgements}

We thank Marla Brunker, from Edanz Group (www.edanzediting.com/ac), for editing a draft of this manuscript.

\section{Authors' contributions}

All authors were involved in the preparation of this manuscript. SS, SN, SW, $M K, H S, K K, T M, A N$, and HS designed the study. SN, SW, KK, TM, AN, and HS analyzed the preoperative data. SS, SN, SW, and II analyzed the surgical and pathological findings. Postoperative follow-up and data analysis were conducted by SS, SN, and SW. SN, SW, II, and HS revised the manuscript. All authors read and approved the final manuscript.

\section{Funding}

The authors declare no financial support

\section{Availability of data and materials}

All data generated or analyzed in the current article are available from the corresponding author on reasonable request.

Ethics approval and consent to participate Not applicable.

\section{Consent for publication}

Written informed consent for publication was obtained from the patient.

\section{Competing interests}

The authors declare that they have no competing interests.

\section{Author details}

'Department of Gastroenterological Surgery, Nagano Red Cross Hospital, 5-22-1, Wakasato, Nagano-shi, Nagano 380-8582, Japan. ${ }^{2}$ Department of Gastroenterology, Nagano Red Cross Hospital, Nagano, Japan. ${ }^{3}$ Department of Pathology, Nagano Red Cross Hospital, Nagano, Japan. 
Received: 20 September 2019 Accepted: 17 December 2019

Published online: 26 December 2019

\section{References}

1. Llovet JM, Bustamante J, Castells A, Vilana R, Ayuso Mdel C, Sala M, et al. Natural history of untreated nonsurgical hepatocellular carcinoma: rationale for the design and evaluation of therapeutic trials. Hepatology. 1999;29(1):62-7.

2. Nouso K, Miyahara K, Uchida D, Kuwaki K, Izumi N, Omata M, et al. Effect of hepatic arterial infusion chemotherapy of 5-fluorouracil and cisplatin for advanced hepatocellular carcinoma in the Nationwide Survey of Primary Liver Cancer in Japan. Br J Cancer. 2013;109(7):1904-7.

3. EASL. Clinical Practice Guidelines: management of hepatocellular carcinoma. J Hepatol. 2018:69(1):182-236.

4. Heimbach JK, Kulik LM, Finn RS, Sirlin CB, Abecassis MM, Roberts LR, et al. AASLD guidelines for the treatment of hepatocellular carcinoma. Hepatology. 2018;67(1):358-80.

5. Cheng AL, Kang YK, Chen Z, Tsao CJ, Qin S, Kim JS, et al. Efficacy and safety of sorafenib in patients in the Asia-Pacific region with advanced hepatocellular carcinoma: a phase III randomised, double-blind, placebocontrolled trial. Lancet Oncol. 2009;10(1):25-34.

6. Llovet JM, Ricci S, Mazzaferro V, Hilgard P, Gane E, Blanc JF, et al. Sorafenib in advanced hepatocellular carcinoma. N Engl J Med. 2008:359(4):378-90.

7. Bruix J, Raoul JL, Sherman M, Mazzaferro V, Bolondi L, Craxi A, et al. Efficacy and safety of sorafenib in patients with advanced hepatocellular carcinoma: subanalyses of a phase III trial. J Hepatol. 2012;57(4):821-9.

8. Kudo M, Finn RS, Qin S, Han KH, Ikeda K, Piscaglia F, et al. Lenvatinib versus sorafenib in first-line treatment of patients with unresectable hepatocellular carcinoma: a randomised phase 3 non-inferiority trial. Lancet. 2018; 391(10126):1163-73.

9. Curtit E, Thiery-Vuillemin A, Nguyen T, Heyd B, Pivot X, Di Martino V, et al. Complete histologic response induced by sorafenib in advanced hepatocellular carcinoma: a case report. J Clin Oncol. 2011;29(12):e330-2.

10. Kermiche-Rahali S, Di Fiore A, Drieux F, Di Fiore F, Francois A, Scotte M. Complete pathological regression of hepatocellular carcinoma with portal vein thrombosis treated with sorafenib. World J Surg Oncol. 2013;11(1):171

11. Takano M, Kokudo T, Miyazaki Y, Kageyama Y, Takahashi A, Amikura K, et al. Complete response with sorafenib and transcatheter arterial chemoembolization in unresectable hepatocellular carcinoma. World J Gastroenterol. 2016;22(42):9445-50

12. Kim DH, Cho E, Cho SB, Choi SK, Kim S, Yu J, et al. Complete response of hepatocellular carcinoma with right atrium and pulmonary metastases treated by combined treatments (a possible treatment effect of natural killer cell): a case report and literature review. Medicine (Baltimore). 2018:97(42):e12866.

13. Shinoda M, Kishida N, Itano O, Ei S, Ueno A, Kitago M, et al. Long-term complete response of advanced hepatocellular carcinoma treated with multidisciplinary therapy including reduced dose of sorafenib: case report and review of the literature. World J Surg Oncol. 2015:13:144.

14. Park JG, Park SY, Lee HW. Complete remission of advanced hepatocellular carcinoma by radiofrequency ablation after sorafenib therapy. World J Gastroenterol. 2015;21(8):2568-72.

15. Kitajima T, Hatano E, Mitsunori Y, Taura K, Fujimoto $Y$, Mizumoto M, et al. Complete pathological response induced by sorafenib for advanced hepatocellular carcinoma with multiple lung metastases and venous tumor thrombosis allowing for curative resection. Clin J Gastroenterol. 2015;8(5):300-5

16. Katafuchi E, Takami Y, Wada Y, Tateishi M, Ryu T, Mikagi K, et al. Long-term maintenance of complete response after sorafenib treatment for multiple lung metastases from hepatocellular carcinoma. Case Rep Gastroenterol. 2015;9(2):285-90

17. Oh YJ, Park YM, Kim BH, Kim MJ, Cho JH, Cha CW, et al. A case of hepatocellular carcinoma with pulmonary metastases treated successfully with a combination of repeated hepatic arterial infusion epirubicin and cisplatin chemotherapy and systemic low-dose infusion of 5-fluorouracil. Gut and liver. 2009;3(4):343-8

18. Kurahashi $S$, Sano $T$, Natsume $S$, Senda $Y$, Yamaura $H$, Inaba $Y$, et al. Surgical treatment after hepatic arterial infusion chemotherapy for hepatocellular carcinoma extending into the right atrium. Surgical case reports. 2015;1(1):47

19. Kogure T, Iwasaki T, Ueno Y, Kanno N, Fukushima K, Yamagiwa Y, et al. Complete remission of a case of hepatocellular carcinoma with tumor invasion in inferior vena cava and with pulmonary metastasis successfully treated with repeated arterial infusion chemotherapy.

Hepatogastroenterology. 2007;54(79):2113-6.

20. Kim YG, Eun JR, Kim TN, Lee HJ, Kim JW, Chang JC, et al. Pathological complete remission of advanced hepatocellular carcinoma with main portal vein tumor thrombosis by hepatic arterial infusion chemotherapy. Gut and liver. 2010;4(2):266-9.

21. Lin CC, Hung CF, Chen WT, Lin SM. Hepatic arterial infusion chemotherapy for advanced hepatocellular carcinoma with portal vein thrombosis: impact of early response to 4 weeks of treatment. Liver cancer. 2015;4(4):228-40.

22. Lai YC, Shih CY, Jeng CM, Yang SS, Hu JT, Sung YC, et al. Hepatic arterial infusion chemotherapy for hepatocellular carcinoma with portal vein tumor thrombosis. World J Gastroenterol. 2003:9(12):2666-70.

23. Ando E, Tanaka M, Yamashita F, Kuromatsu R, Yutani S, Fukumori K, et al. Hepatic arterial infusion chemotherapy for advanced hepatocellular carcinoma with portal vein tumor thrombosis: analysis of 48 cases. Cancer. 2002;95(3):588-95.

24. Moriguchi M, Aramaki T, Nishiofuku H, Sato R, Asakura K, Yamaguchi K, et al. Sorafenib versus hepatic arterial infusion chemotherapy as initial treatment for hepatocellular carcinoma with advanced portal vein tumor thrombosis. Liver cancer. 2017;6(4):275-86.

25. Nakano M, Niizeki T, Nagamatsu H, Tanaka M, Kuromatsu R, Satani M, et al. Clinical effects and safety of intra-arterial infusion therapy of cisplatin suspension in lipiodol combined with 5-fluorouracil versus sorafenib, for advanced hepatocellular carcinoma with macroscopic vascular invasion without extra-hepatic spread: a prospective cohort study. Molecular and clinical oncology. 2017;7(6):1013-20.

26. Kudo M, Ueshima K, Yokosuka O, Ogasawara S, Obi S, Izumi N, et al. Sorafenib plus low-dose cisplatin and fluorouracil hepatic arterial infusion chemotherapy versus sorafenib alone in patients with advanced hepatocellular carcinoma (SILIUS): a randomised, open label, phase 3 trial. The Lancet Gastroenterology \& hepatology. 2018;3(6):424-32.

27. Niizeki T, Sumie S, Torimura T, Kurogi J, Kuromatsu R, Iwamoto H, et al. Serum vascular endothelial growth factor as a predictor of response and survival in patients with advanced hepatocellular carcinoma undergoing hepatic arterial infusion chemotherapy. J Gastroenterol. 2012;47(6):686-95.

28. Rapisarda A, Melillo G. Role of the hypoxic tumor microenvironment in the resistance to anti-angiogenic therapies. Drug Resist Updat. 2009;12(3):74-80

29. Sakamaki A, Kamimura K, Abe S, Tsuchiya A, Takamura M, Kawai H, et al. Spontaneous regression of hepatocellular carcinoma: a mini-review. World J Gastroenterol. 2017;23(21):3797-804.

\section{Publisher's Note}

Springer Nature remains neutral with regard to jurisdictional claims in published maps and institutional affiliations.

Ready to submit your research? Choose BMC and benefit from:

- fast, convenient online submission

- thorough peer review by experienced researchers in your field

- rapid publication on acceptance

- support for research data, including large and complex data types

- gold Open Access which fosters wider collaboration and increased citations

- maximum visibility for your research: over $100 \mathrm{M}$ website views per year

At BMC, research is always in progress.

Learn more biomedcentral.com/submission 